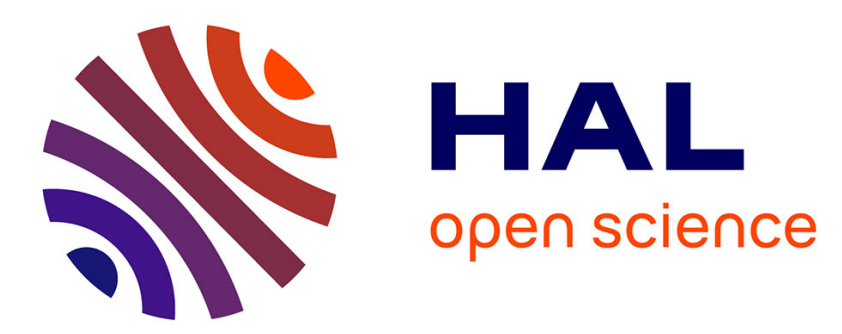

\title{
Savings behavior with imperfect capital markets: when hyperbolic discounting leads to discontinuous strategies
}

Bertrand Wigniolle

\section{To cite this version:}

Bertrand Wigniolle. Savings behavior with imperfect capital markets: when hyperbolic discounting leads to discontinuous strategies. Economics Letters, 2012, 116 (2), pp.186-189. 10.1016/j.econlet.2012.02.014 . halshs-00684210

\section{HAL Id: halshs-00684210 \\ https://shs.hal.science/halshs-00684210}

Submitted on 30 Mar 2012

HAL is a multi-disciplinary open access archive for the deposit and dissemination of scientific research documents, whether they are published or not. The documents may come from teaching and research institutions in France or abroad, or from public or private research centers.
L'archive ouverte pluridisciplinaire HAL, est destinée au dépôt et à la diffusion de documents scientifiques de niveau recherche, publiés ou non, émanant des établissements d'enseignement et de recherche français ou étrangers, des laboratoires publics ou privés. 


\title{
Savings behavior with imperfect capital markets: when hyperbolic discounting leads to discontinuous strategies
}

\author{
Bertrand Wigniolle*
}

November 4, 2011

*Paris School of Economics and University of Paris 1 Panthéon-Sorbonne. Address: C.E.S., Maison des Sciences Economiques, 106-112, boulevard de l'hôpital, 75647 Paris Cedex 13, France. Tel: +33 (0)1 440781 98. Fax: +33 (0)1 440782 31. Email : wignioll@univ-paris1.fr. 


\begin{abstract}
The joint assumptions of quasi-hyperbolic discounting and imperfect capital market lead to non-convexities in selves' objective functions that may imply discontinuous equilibrium strategies. If this property was early recognized, authors generally take assumptions on parameters that allow to avoid the problem. In contrast to the literature, I choose to give a full characterization of these strategies in a simple model that allows a complete determination of savings behavior. Savings function undergoes jumps and nonmonotonicities when the incomes or the interest rates reach threshold values. These "anomalies" may exist even for "reasonable" parameters values.
\end{abstract}

JEL classification: D03, D91

Keywords: quasi-hyperbolic discounting, no-borrowing constraint, discontinuous strategies. 


\section{Introduction}

When the assumption of hyperbolic discounting is associated with imperfect capital markets, it is possible that selves face a non-convex problem that may generate discontinuous equilibrium strategies (see Laibson (1997)). Therefore, authors generally adopt some restrictions on parameters that allow to eliminate this problem. Only Harris and Laibson (2002) deal with this question. They present numerical simulations that show that irregularities in the consumption function tend to disappear if the bias for the present is weak and the intertemporal elasticity of substitution is low.

The aim of this paper is to challenge Harris and Laibson's conclusions, Firstly, their results were obtained by numerical simulations. One aim is to go beyond these results, by a detailed study of consumption and savings behaviors in a simple framework that allows a complete characterization of strategies. Secondly, hasty conclusions should not be taken from Harris and Laibson's results. If the bias for the present is weak and the intertemporal elasticity of substitution is low, the behavior with hyperbolic discounting is very close to the behavior with exponential discounting. When hyperbolic discounting does not matter, it is not surprising to find that discontinuities vanish. On the other hand, if hyperbolic discounting actually modifies agents' behaviors, it is also associated with discontinuous equilibrium strategies. Thirdly, discontinuous equilibrium strategies can be interesting, as they provide an example of a behavior that qualitatively differs from the standard model. In the model with exponential discounting, consumption behaviors are continuous functions of the different parameters: interest rates, labor incomes, etc. With hyperbolic discounting, a continuous change of one parameter can induce a jump in consumption and savings. Therefore, the two models of consumption and savings are not observationally equivalent.

Section 2 presents the model. Section 3 gives a formal characterization of all possible solutions. Section 4 gives an overall picture of the results and points out some consequences on savings functions. Section 5 concludes.

\section{The model}

\subsection{Basic assumptions}

The framework is a three-period quasi-hyperbolic discounting model. In period 1 , self 1 preferences are given by the utility function:

$$
U(c, d, e)=u(c)+\beta \delta u(d)+\beta \delta^{2} u(e), \text { with } u(x)=\frac{x^{1-\frac{1}{\sigma}}}{1-\frac{1}{\sigma}}
$$


and $\sigma>0 . c, d$ and $e$ are respectively the consumption levels in period 1,2 and 3 .

In period 2, self 2's preferences are given by the utility function:

$$
u(d)+\beta \delta u(e)
$$

The budget constraints are:

$$
\begin{aligned}
c+x & =A \\
d+y & =B+R x \\
e & =C+R y
\end{aligned}
$$

$A, B$ and $C$ are respectively the income amounts earned in period 1,2 and 3. $x$ and $y$ are first and second period savings. $R$ is the factor of interest. $\beta$ and $\delta$ are two positive coefficients not greater than $1 . \delta$ is the usual discount parameter and $\beta$ is the bias for the present. A self-control problem exists for $\beta<1$.

Capital markets are imperfect: $x \geq 0$ and $y \geq 0$. These constraints mean that the agent cannot be indebted. $x$ is the decision variable of self 1 and $y$ the decision variable of self 2 . The sequence of decisions results from the equilibrium of the game between selves 1 and 2, in which self 1 plays first (sophisticated behavior). Self 2 chooses $y$, in taking $x$ as given. Self 1 chooses $x$, taking into account the best response function of self 2 .

\subsection{Self 2's behavior}

The program of self 2 is:

$$
\left\{\begin{array}{l}
\max _{(y)} u(B+R x-y)+\beta \delta u(C+R y) \\
\text { s. t. } y \geq 0
\end{array}\right.
$$

Defining the threshold

$$
\tilde{x} \equiv \frac{1}{R}\left[\frac{C}{(R \beta \delta)^{\sigma}}-B\right]
$$

the best response function of self 2 is:

$$
Y(x)=\left\{\begin{array}{cl}
\frac{(R \beta \delta)^{\sigma}(B+R x)-C}{R+(R \beta \delta)^{\sigma}} & \text { if } x \geq \tilde{x} \\
0 & \text { if } x \leq \tilde{x}
\end{array}\right.
$$

In the case $C \leq B(R \beta \delta)^{\sigma}, y$ is always non-negative, even if $x=0$. The optimal choice of $y$ is not constrained by the constraint $y \geq 0$. In the opposite 
case $C>B(R \beta \delta)^{\sigma}$, it is possible that the constraint is binding for self 2 : $y=0$. As a consequence, the choice of $x$ by self 1 can determine if self 2 is constrained or not.

\subsection{The case $C \leq B(R \beta \delta)^{\sigma}$}

In this case, $y$ is non-negative whatever the choice of $x$. The optimal behavior of self 1 derives from:

$$
\left\{\begin{array}{l}
\max _{(x)} u(A-x)+\beta \delta u(B+R x-Y(x))+\beta \delta u(C+R Y(x)) \\
\text { s. t. } x \geq 0
\end{array}\right.
$$

Defining

$$
\begin{aligned}
Z & \equiv \beta \delta \frac{\left[1+\delta^{\sigma}(R \beta)^{\sigma-1}\right]}{\left[1+R^{\sigma-1}(\beta \delta)^{\sigma}\right]^{1-1 / \sigma}} \\
\hat{x} & \equiv \frac{R^{\sigma} Z^{\sigma} A-B-\frac{C}{R}}{R+R^{\sigma} Z^{\sigma}}
\end{aligned}
$$

The solution is: If $R^{\sigma} Z^{\sigma} A>B+C / R, x=\hat{x}$; if $R^{\sigma} Z^{\sigma} A \leq B+C / R, x=0$.

Using the optimal value of $x$ (either $\hat{x}$ given by (6) or 0 ), it is possible to find the equilibrium value of $y$ given by $Y(x)$ (cf. equation (3)).

\section{Resolution in the case $C>B(R \beta \delta)^{\sigma}$}

\subsection{The non-concavity of self 1's objective function}

The case $C>B(R \beta \delta)^{\sigma}$ is more interesting as it is possible that the optimal choice of $y$ is positive or 0 , depending on the choice of $x$. The problem of the non-concavity of the objective function of self 1 may occur. This function depends on the variable $x$ according to

$$
\phi(x) \equiv u(A-x)+\beta \delta u(B+R x-Y(x))+\beta \delta^{2} u(C+R Y(x))
$$

Taking the derivative with respect to $x($ with $x \neq \tilde{x})$,

$$
\phi^{\prime}(x)=u^{\prime}(c)+R \beta \delta u^{\prime}(d)+Y^{\prime}(x) \beta \delta\left[-u^{\prime}(d)+R \delta u^{\prime}(e)\right]
$$

If the consumer had no problem of self control $(\beta=1)$, the expression $\left[-u^{\prime}(d)+R \delta u^{\prime}(e)\right]$ would correspond to the derivative of self 2's objective function. This term cancels out $\left(-u^{\prime}(d)+R \delta u^{\prime}(e)=0\right)$ along the best 
response function of self 2 . This is a simple consequence of the envelope theorem. The derivative of self 1 's objective function (7) remains continuous.

In the case $\beta<1$, there is a discrepancy between the objective functions of self 1 and self 2 . From the first order condition of self $2,-u^{\prime}(d)+\beta R \delta u^{\prime}(e)=$ 0 , which implies $-u^{\prime}(d)+R \delta u^{\prime}(e)>0$. Moreover, for $x=\tilde{x}, Y^{\prime}(x)$ is discontinuous: $Y^{\prime}(x)=0$ to the left of $\tilde{x}$, and $Y^{\prime}(x)>0$ to the right.

The consequence of this analysis is that $\phi^{\prime}(x)$ is discontinuous at the point $\tilde{x}$, with a higher value to the right of $\tilde{x}$. It is then possible that $\phi$ admits two local maxima. $\phi$ is concave on each interval $(0, \tilde{x})$ and $(\tilde{x},+\infty)$ and continuous, but the derivative is discontinuous in $\tilde{x}$. In the case of two local maxima, the two values of $\phi$ must be compared.

\subsection{Self 1's objective function}

$\phi$ is studied on the interval $(\tilde{x},+\infty)$ for which $Y(x)$ is positive, and on $(0, \tilde{x})$ for which $Y(x)$ cancels out. The parameters $K, L$ and $\check{x}$ are defined as:

$$
\begin{aligned}
K & \equiv \frac{C}{R^{\sigma} Z^{\sigma}(R \beta \delta)^{\sigma}}\left[1+R^{\sigma-1} Z^{\sigma}+R^{\sigma-1}(\beta \delta)^{\sigma}\right] \\
L & \equiv \frac{C}{(R \beta \delta)^{2 \sigma}}\left[1+R^{\sigma-1}(\beta \delta)^{\sigma}\right] \\
\check{x} & \equiv \frac{(R \beta \delta)^{\sigma} A-B}{R+(R \beta \delta)^{\sigma}}
\end{aligned}
$$

Lemma 1 ( $\phi$ on $(\tilde{x},+\infty))$ If $A+\frac{B}{R}>K$, $\phi$ has a local maximum $\hat{x} \in$ $(\tilde{x},+\infty)$ given by (6), with $Y(\hat{x})>0$. If $A+\frac{B}{R} \leq K, \phi$ is decreasing on $(\tilde{x},+\infty)$.

Lemma 2 ( $\phi$ on $(0, \tilde{x})$ ) If $(R \beta \delta)^{\sigma} A \geq B$, two cases exist: if $A+\frac{B}{R}<L, \phi$ has a local maximum $\check{x} \in(0, \tilde{x})$ given by (10), with $Y(\check{x})=0$; If $A+\frac{B}{R} \geq L$, $\phi$ is increasing on $(0, \tilde{x})$. If $(R \beta \delta)^{\sigma} A<B, \phi$ is decreasing on the interval $(0, \tilde{x})$.

\subsection{The solution of the game}

From (8) and (9), it follows that $K<L$ as $\beta<1$. Therefore, three cases must be studied separately.

\subsubsection{Case $A+B / R \leq K$}

The intertemporal wealth of the agent on the two first periods is weak $(A+B / R \leq K)$, and the third period income is high $\left(C>B(R \beta \delta)^{\sigma}\right)$. 
The solution is always associated with $y=0 . x$ can be null or equal to $\breve{x}$, depending on the two first period incomes. The absence of concavity has no consequence, and the optimal solution for self 1 is always reached on the interval $(0, \tilde{x})$.

Proposition 1 If $A+B / R \leq K$, the solution of the game between selves 1 and 2 is:

- if $(R \beta \delta)^{\sigma} A \geq B, x=\check{x}$ given by (10) and $y=0$;

- if $(R \beta \delta)^{\sigma} A<B, x=0$ and $y=0$.

\subsubsection{Case $A+B / R \geq L$}

The intertemporal wealth on the two first periods is high $(A+B / R \geq L)$. The optimal solution of self 1 leads to the interior solution $x=\hat{x}$ and $y=Y(\hat{x})$. The absence of concavity has no consequence, and the optimal solution for self 1 is always reached on the interval $(\tilde{x},+\infty)$.

Proposition 2 If $A+B / R \geq L$, the solution is: $x=\hat{x}$ and $y=Y(\hat{x})$.

\subsubsection{Case $K<A+B / R<L$}

The objective function $\phi$ of self 1 has two local maxima, one in each interval $(0, \tilde{x})$ and $(\tilde{x},+\infty)$. Therefore, it is necessary to compare the utility levels at these two maxima.

First case: $(R \beta \delta)^{\sigma} A \geq B$. In this case, $\check{x}$ (given by (10)) is a local maximum of $\phi$ on $(0, \tilde{x})$, and $\hat{x}$ (given by (6)) is another one on $(\tilde{x},+\infty)$. The comparison of the corresponding utility levels allows to obtain the results:

Proposition 3 Assume that $K<A+B / R<L$ and $(R \beta \delta)^{\sigma} A \geq B$. There exists $M \in(K, L)$ such that,

- if $K<A+B / R<M$, the solution is $x=\check{x}$ and $y=0$.

- if $M<A+B / R<L$, the solution is $x=\hat{x}$ and $y=Y(\hat{x})$

- if $A+B / R=M$, self 1 is indifferent between $x=\check{x}$ and $y=0$, or $x=\hat{x}$ and $y=Y(\hat{x})$. 
This proposition shows that for $(R \beta \delta)^{\sigma} A \geq B$, the line $A+B / R=M$ is the pertinent frontier that separates the two types of savings behaviors: $x=\hat{x}$ and $y=Y(\hat{x})$ or $x=\check{x}$ and $y=0$. A consequence of this result is that, if $A+B / R$ is close to $M$, a small change in one parameter $(A$, or $B$ or $R$ ) can have a dramatic effect on savings behavior: $y$ can jump from 0 to $Y(\hat{x})$, and $x$ can jump from $\check{x}$ to $\hat{x}$.

Second case: $(R \beta \delta)^{\sigma} A<B$. In this case, $x=0$ is a local maximum of $\phi$ on $(0, \tilde{x})$, and $\hat{x}$ (given by $(6))$ is another one on $(\tilde{x},+\infty)$. The comparison of the corresponding utility levels gives the results:

Proposition 4 Assume that $(R \beta \delta)^{\sigma} A<B$.

1. If $M \leq A+B / R<L$, the solution is: $x=\hat{x}$ and $y=Y(\hat{x})$.

2. If $K<A+B / R<M$. There exists a decreasing function $\Delta(A)$ defined on $\left[A_{H}, A_{I}\right]$, with

$$
\begin{aligned}
A_{H} & =\frac{C}{(R Z)^{\sigma}(R \beta \delta)^{\sigma}}\left[1+R^{\sigma-1}(\beta \delta)^{\sigma}\right], \Delta\left(A_{H}\right)=\frac{C}{(R \beta \delta)^{\sigma}} \\
A_{I} & =\frac{M}{1+R^{\sigma-1}(\beta \delta)^{\sigma}}, \Delta\left(A_{I}\right)=\frac{M(R \beta \delta)^{\sigma}}{1+R^{\sigma-1}(\beta \delta)^{\sigma}}
\end{aligned}
$$

such that,

- if $B<\Delta(A)$, the solution is $x=0$ and $y=0$.

- if $B>\Delta(A)$, the solution is $x=\hat{x}$ and $y=Y(\hat{x})$.

- if $B=\Delta(A)$, self 1 is indifferent between these two solutions.

When the intertemporal wealth of the consumer during the two first periods is high enough $(A+B / R \geq M)$, the optimal choice for self 1 is $x=\hat{x}$ (and $y=Y(\hat{x})$ for self 2 ). When $A+B / R<M$, the choice of self 1 remains $x=\hat{x}$ only if $B$ is high enough with respect to $A(B>\Delta(A))$. In the converse case, self 1 chooses $x=0$ and self $2 y=0$. 


\section{Final results and consequences}

\subsection{An overall picture of the consumer's choice}

In taking the value of $C$ as given, an overall characterization of the consumer's savings choices is obtained. Four cases may arise, depending on $A$ and $B$ :

$$
\begin{aligned}
Z_{1}= & \left\{(A, B) \text { s.t. }(R \beta \delta)^{\sigma} A>B \text { and } A+B / R<M\right\} \\
Z_{2}= & \left\{(A, B) \text { s. t. }(R \beta \delta)^{\sigma} A<B, B<C(R \beta \delta)^{-\sigma},\right. \\
& \left.A+B / R<M \text { and } B<\Delta(A) \text { for } A \in\left(A_{H}, A_{I}\right)\right\} \\
Z_{3}= & \left\{(A, B) \text { s.t. } A>A_{H}, \quad(R Z)^{\sigma} A>B+C / R, B>\Delta(A) \text { for } A \in\left(A_{H}, A_{I}\right),\right. \\
& \left.A+B / R>M \text { for } A>A_{I}\right\} \\
Z_{4}= & \left\{(A, B) \text { s. t. } B>C(R \beta \delta)^{-\sigma} \text { and }(R Z)^{\sigma} A>B+C / R\right\}
\end{aligned}
$$

The preceding results give the consumer's choice in each set ${ }^{1}$ :

- in $Z_{1}: x=\check{x}$ given by $(10)$ and $y=0$.

- in $Z_{2}: x=0$ and $y=0$.

- in $Z_{3}: x=\hat{x}$ given by $(6)$ and $y=Y(\hat{x})$ given by (3).

- in $Z_{4}: x=0$ and $y=Y(0)$ given by $(3)$.

Figure (1) represents the characterization of the different zones of the plane $(A, B)$. The frontiers are in bold lines. The figure is obtained for the following parameters values, in line with the calibration of Harris and Laibson (2002): $\beta=0.7, \delta=0.9571, R=1.0375, C=6$ and $\sigma=0.99$. The value of $\sigma$ is a controversial question. A value close to one corresponds to the one retained in calibrated macroeconomic models. Some recent works (see e. g. Favero 2005) support such an assumption.

\subsection{Consequences on savings function}

The assumption of quasi-hyperbolic discounting can have strong consequences on savings behaviors, when self 1's objective function is not concave. The optimal strategy of self 1 can be discontinuous with respect to his income. Different illustrations are provided.

The value of $C$ is fixed to $C=6$. The impact of $A$ and $B$ on the amount of savings $x$ is studied. In Figure (2), $x$ is represented as a function of $B$

\footnotetext{
${ }^{1}$ The frontiers have not been included in the 4 sets as a point on a frontier may make self 1 indifferent between 2 different strategies.
} 
for two different values of $A: A=16$ and $A=11.13 .^{2}$. With $A=16$, starting from $B=0$ and increasing $B$, the incomes $\{A, B\}$ of the consumer first belong to zone 1 , and successively reach zone 3 and 4 (cf. Figure (1)). When $B$ reaches the value such that $M=A+B / R, x$ jumps from $x=\check{x}$ to $x=\hat{x}>\check{x}$. The slope of the savings function goes from -0.58 to -0.41 .

With $A=11.13$, as $B$ increases, the incomes of the consumer belong to zone 1 at the beginning, and successively reach zone 2, 3 and 4 (cf. Figure $(1))$. In zone $1, x=\check{x}$ and decreases with $B$ till 0 . In zone $2, x$ remains equal to 0 . When crossing the frontier $\Delta(A), x$ jumps from 0 to a positive value $\hat{x}$. In zone $3, x=\hat{x}$ and decreases with $B$ till 0 . In zone $4, x$ remains equal to 0 .

The effect of $A$ on savings for a given value of $B$ is simulated for $B=4$ (cf. Figure (3)). Starting from $A=0$ and increasing $A$, consumer incomes cross zones 2, 1 and 3 (cf. Figure (1)). In zone $2, x=0$. In zone $1, x=\check{x}$ and increases with $A$. When $A$ reaches the value such that $M=A+B / R, x$ jumps from $x=\check{x}$ to $x=\hat{x}>\check{x}$. The slope of the savings function goes from 0.40 to 0.57 .

In these simple examples, the evolution of savings $x$ with respect to $A$ and $B$ has been considered. It is possible to make the same study with the other parameters. For instance, a jump of savings can also be obtained through a change in the interest factor $R$.

\footnotetext{
${ }^{2}$ The value 11.13 corresponds to $\left(A_{H}+A_{I}\right) / 2$.
} 


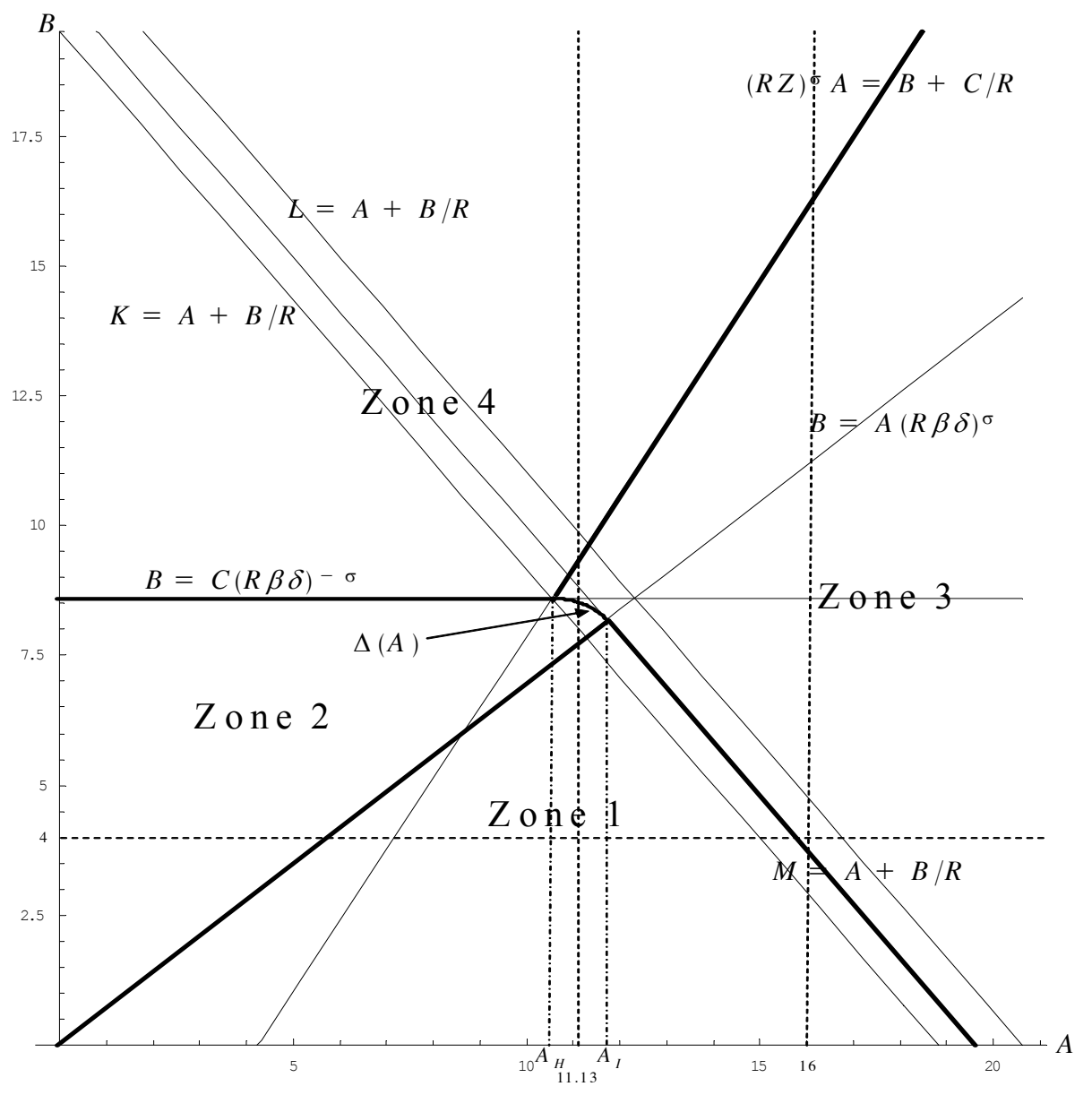

Figure 1: the different zones

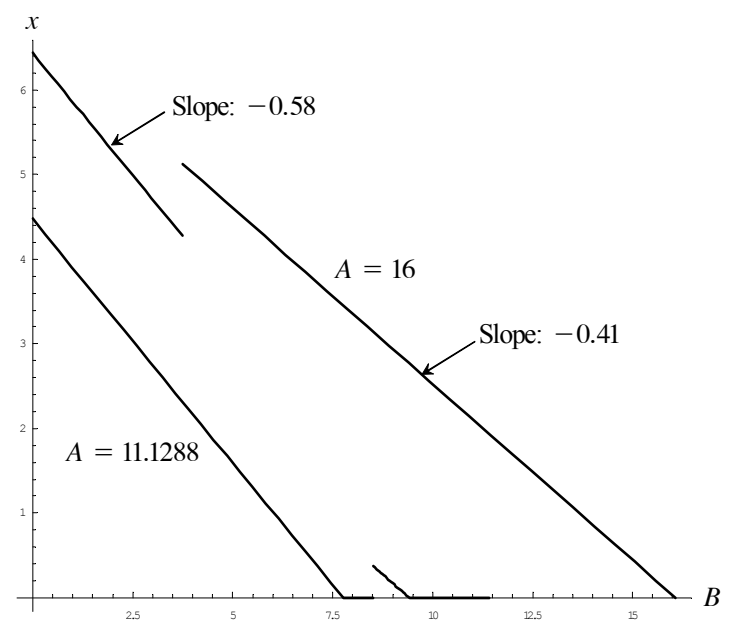

Figure 2: savings $x$ with respect to $B$

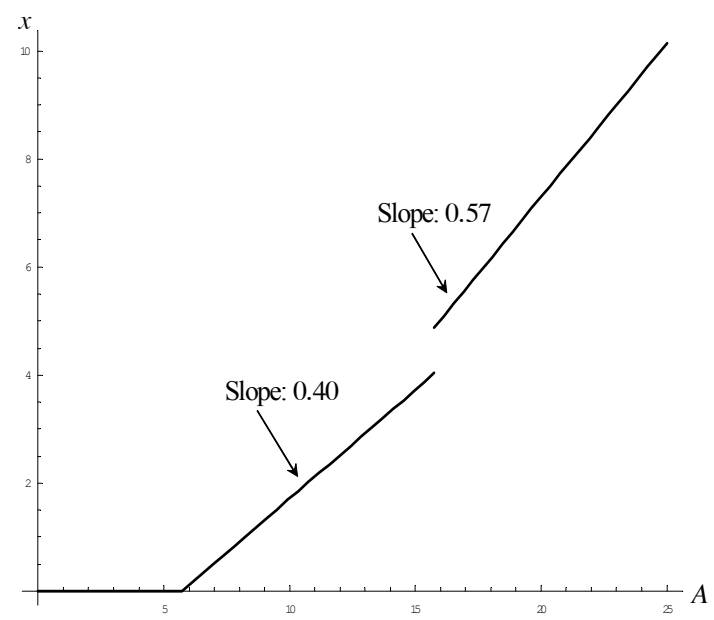

Figure 3: savings $x$ with respect to $A$ 


\section{Conclusion}

Our numerical simulations show that savings functions under quasi hyperbolic discounting present significative differences with respect to the standard case, when discontinuous strategies are taken into account. Firstly, savings functions may undergo upward jumps, resulting from an increase in incomes. Secondly, after these jumps, the slope of savings with respect to income goes up. These theoretical results may have important consequences for the empirical analysis of savings. If agents behave as predicted by the theory, savings functions should be estimated through functional forms able to deal with these jumps and changes in slopes.

\section{References}

[1] Favero, C., 2005. Consumption, Wealth, the Elasticity of Intertemporal Substitution and Long-Run Stock Market Returns. Working Papers 291, IGIER, Bocconi University.

[2] Harris, C., Laibson, D., 2002. Hyperbolic discounting and consumption, in: Dewatripont, M., Hansen, L.P., Turnovsky, S. (Eds.), Advances in Economics and Econometrics: Theory and Applications, Eighth World Congress, (1) pp. 258-298.

[3] Laibson, D., 1997. Golden Eggs and Hyperbolic Discounting. Quart. J. Econ. 112(2), 443-477. 


\section{Supplementary data}

1. Proof of lemma 1: $\phi$ on $(\tilde{x},+\infty)$

First the solution is assumed to be such that $y>0$, or $(B+R x)(R \beta \delta)^{\sigma}>$ $C$. The value of $x$ chosen by self 1 results from program (4). The solution $x$ can either be a positive value given by (6), or $x=0$. As $C>B(R \beta \delta)^{\sigma}$, the solution $x=0$ is not compatible with the assumption $y>0$, because $x=0$ implies $y=0$. Therefore, to obtain the case $y>0$ as a solution, it is necessary that $R^{\sigma} Z^{\sigma} A>B+C / R$, and $x=\hat{x}$. Moreover, to obtain a positive value for $y=Y(\hat{x})$, it is necessary that $\hat{x}>\tilde{x}$. Using (2) and (6), the inequality $\hat{x}>\tilde{x}$ leads to:

$$
A+\frac{B}{R}>\frac{C}{R^{\sigma} Z^{\sigma}(R \beta \delta)^{\sigma}}\left[1+R^{\sigma-1} Z^{\sigma}+R^{\sigma-1}(\beta \delta)^{\sigma}\right] \equiv K
$$

When $C>B(R \beta \delta)^{\sigma}$, as $\tilde{x}>0$ the inequality $\hat{x}>\tilde{x}$ is stronger than $\hat{x}>0$. Therefore, if $A+B / R>K$, the condition $R^{\sigma} Z^{\sigma} A>B+C / R$ holds.

To sum up, if $A+B / R>K$, self 1's objective function has a local maximum $\hat{x} \in(\tilde{x},+\infty)$ given by (6), with $Y(\hat{x})>0$. If $A+B / R \leq K$, self 1 's objective function is decreasing on $(\tilde{x},+\infty)$, as the maximum of the objective function is obtained for a value of $x$ smaller than $\tilde{x}$.

2. Proof of lemma 2: $\phi$ on $(0, \tilde{x})$

This type of solution is such that $y=0$. In this case, self 1 's program is given by:

$$
\left\{\begin{array}{l}
\max _{(x)} \frac{1}{1-1 / \sigma}(A-x)^{1-1 / \sigma}+\frac{\beta \delta}{1-1 / \sigma}(B+R x)^{1-1 / \sigma}+\frac{\beta \delta^{2}}{1-1 / \sigma}(C)^{1-1 / \sigma} \\
\text { s. t. } x \geq 0
\end{array}\right.
$$

The solution is:

- If $(R \beta \delta)^{\sigma} A \geq B$

$$
x=\frac{(R \beta \delta)^{\sigma} A-B}{R+(R \beta \delta)^{\sigma}} \equiv \check{x}
$$

- If $(R \beta \delta)^{\sigma} A<B, x=0$. The objective function is decreasing on the interval $(0, \tilde{x})$, as the maximum is reached for a negative value of $x$.

In the case $(R \beta \delta)^{\sigma} A \geq B$, the value of $x$ given by (10) is admissible only if it belongs to $(0, \tilde{x})$. In the converse case, $y$ should be positive. Using (10) and (2), the inequality $\check{x}<\tilde{x}$ leads to

$$
A+\frac{B}{R}<\frac{C}{(R \beta \delta)^{2 \sigma}}\left[1+R^{\sigma-1}(\beta \delta)^{\sigma}\right] \equiv L
$$


To sum up, if $(R \beta \delta)^{\sigma} A \geq B$ and if $A+B / R<L$, self 1's objective function has a local maximum $\check{x} \in(0, \tilde{x})$ given by $(10)$, with $Y(\check{x})=0$. If $A+B / R \geq L$, it means that self 1 's objective function is increasing on the interval $(0, \tilde{x})$, as the maximum is obtained for a value of $x$ greater than $\tilde{x}$. If $(R \beta \delta)^{\sigma} A<B$, self 1's objective function is decreasing on the interval $(0, \tilde{x})$.

3. Proof of proposition 1: solution in the case $A+B / R \leq K$

If $A+B / R \leq K$, it is clear that $A+B / R<L$. From lemma (1), self 1 's objective function is decreasing on the interval $(\tilde{x},+\infty)$. On the interval $(0, \tilde{x})$, from lemma (2), two cases may happen: If $(R \beta \delta)^{\sigma} A \geq B, \phi$ reaches a local maximum in $x=\check{x}>0$ given by (10). If $(R \beta \delta)^{\sigma} A<B, \phi$ is decreasing on the interval $(0, \tilde{x})$. From these properties, the result follows.

4. Proof of proposition 2: solution in the case $A+B / R \geq L$

If $A+B / R \geq L$, it is clear that $A+B / R>K$. Moreover, combining the two inequalities

$$
\begin{aligned}
C & >B(R \beta \delta)^{\sigma} \\
A+\frac{B}{R} & \geq \frac{C}{(R \beta \delta)^{2 \sigma}}\left[1+R^{\sigma-1}(\beta \delta)^{\sigma}\right]
\end{aligned}
$$

one obtains that $(R \beta \delta)^{\sigma} A>B$. Therefore it is possible to deduce in this case that:

- on the interval $(0, \tilde{x}), \phi$ is increasing on the interval $(0, \tilde{x})$.

- on the interval $(\tilde{x},+\infty), \phi$ has a maximum $\hat{x}$.

Finally, the result is obtained.

5. Proof of proposition 3: solution in the case $K<A+B / R<L$ and $(R \beta \delta)^{\sigma} A \geq B$

In this case, lemmas 1 and 2 show that self 1 's objective function has two local maxima: on $(0, \tilde{x}), \check{x}$ given by $(10)$ is a local maximum, and $Y(\check{x})=$ 0 ; on $(\tilde{x},+\infty), \hat{x}$ given by (6) is another one, with $Y(\hat{x})>0$. To know what is the final choice of self 1 , it is necessary to compare the value of the objective function at these two points $\check{x}$ and $\hat{x}$. When $x=\check{x}$ and $y=0$, the corresponding values for consumption are denoted by $\check{c}, \check{d}$ and $\check{e}$ and the utility level of self 1 is $U(\check{c}, \check{d}, \check{e})$. When $x=\hat{x}$ and $y=Y(\hat{x})$, the corresponding values for consumption are denoted by $\hat{c}, \hat{d}$ and $\hat{e}$ and the utility level of self 1 is $U(\hat{c}, \hat{d}, \hat{e})$. The following lemma allows to do the comparison of utility levels. 
Lemma 3 - The values of $U(\check{c}, \check{d}, \check{e})$ and $U(\hat{c}, \hat{d}, \hat{e})$ are given by

$$
\begin{aligned}
U(\check{c}, \check{d}, \check{e}) & =\frac{\sigma}{\sigma-1}\left(A+\frac{B}{R}\right)^{1-\frac{1}{\sigma}}\left[1+R^{\sigma-1}(\beta \delta)^{\sigma}\right]^{\frac{1}{\sigma}}+\frac{\sigma}{\sigma-1} \beta \delta^{2}(C)^{1-\frac{1}{\sigma}} \\
U(\hat{c}, \hat{d}, \hat{e}) & =\frac{\sigma}{\sigma-1}\left(A+\frac{B}{R}+\frac{C}{R^{2}}\right)^{1-\frac{1}{\sigma}}\left[1+R^{\sigma-1} Z^{\sigma}\right]^{\frac{1}{\sigma}}
\end{aligned}
$$

- Let us define the function $F$ as:

$$
\begin{aligned}
F(\xi)= & \frac{\sigma}{\sigma-1}\left(\xi+\frac{C}{R^{2}}\right)^{1-\frac{1}{\sigma}}\left[1+R^{\sigma-1} Z^{\sigma}\right]^{\frac{1}{\sigma}} \\
& -\frac{\sigma}{\sigma-1}(\xi)^{1-\frac{1}{\sigma}}\left[1+R^{\sigma-1}(\beta \delta)^{\sigma}\right]^{\frac{1}{\sigma}}-\frac{\sigma}{\sigma-1} \beta \delta^{2}(C)^{1-\frac{1}{\sigma}}
\end{aligned}
$$

$F$ is defined in such a way that $F(A+B / R)=U(\hat{c}, \hat{d}, \hat{e})-U(\check{c}, \check{d}, \check{e})$.

$F$ is strictly increasing on $[K, L]$, with $F(K)<0$ and $F(L)>0$.

Proof. The first part of lemma (1) is a straightforward calculation.

The second part is concerned with the study of the function $F(\xi)=$ $U(\hat{c}, \hat{d}, \hat{e})-U(\check{c}, \check{d}, \check{e})$ with $\xi=A+B / R$.

Firstly it is proved that $F(K)<0$. The case $\xi=K$ corresponds to the limit case $\hat{x}=\tilde{x}$ when self 1 's objective function is studied on $(\tilde{x},+\infty)$. From Section 3.1, it is known that the derivative of self 1's objective function is discontinuous at the point $\tilde{x}$, with a higher value to the right of $\tilde{x}$. When $\hat{x}=\tilde{x}$ the value of the derivative on the right is 0 . Therefore, the value of the derivative on the left is negative. This proves that $U(\hat{c}, \hat{d}, \hat{e})<U(\check{c}, \check{d}, \check{e})$ or $F(K)<0$.

Secondly it is proved that $F(L)>0$. The case $\xi=L$ corresponds to the limit case $\check{x}=\tilde{x}$ when self 1 's objective function is studied on $(0, \tilde{x})$. From Section 3.1, it is known that the derivative of self 1's objective function is discontinuous at the point $\tilde{x}$, with a higher value to the right of $\tilde{x}$. When $\check{x}=\tilde{x}$ the value of the derivative on the left is 0 . Therefore, the value of the derivative on the right is positive. This proves that $U(\hat{c}, \hat{d}, \hat{e})>U(\check{c}, \check{d}, \check{e})$ or $F(L)>0$.

Finally, it remains to show that $F^{\prime}(\xi)>0$. The derivative is:

$$
F^{\prime}(\xi)=\left(\xi+\frac{C}{R^{2}}\right)^{-\frac{1}{\sigma}}\left[1+R^{\sigma-1} Z^{\sigma}\right]^{\frac{1}{\sigma}}-(\xi)^{-\frac{1}{\sigma}}\left[1+R^{\sigma-1}(\beta \delta)^{\sigma}\right]^{\frac{1}{\sigma}}
$$

The condition $F^{\prime}(\xi)>0$, after some calculations, can be expressed:

$$
\xi>C \frac{1+R^{\sigma-1}(\beta \delta)^{\sigma}}{R^{\sigma-1}\left[Z^{\sigma}-(\beta \delta)^{\sigma}\right]}
$$


where it is important to note that $Z^{\sigma}-(\beta \delta)^{\sigma}>0$. Indeed, from (5), the condition $Z^{\sigma}>(\beta \delta)^{\sigma}$ is equivalent to:

$$
\vartheta(\beta) \equiv \frac{\left[1+\delta^{\sigma}(R \beta)^{\sigma-1}\right]^{\sigma}}{\left[1+R^{\sigma-1}(\beta \delta)^{\sigma}\right]^{\sigma-1}}>1
$$

For $\beta=1, \vartheta(1)=1+\delta^{\sigma} R^{\sigma-1}>1$. The derivative is

$$
\frac{d \ln [\vartheta(\beta)]}{d \beta}=\frac{\sigma(\sigma-1) \delta^{\sigma} R^{\sigma-1} \beta^{\sigma-2}(1-\beta)}{\left(1+\delta^{\sigma} \beta^{\sigma-1} R^{\sigma-1}\right)\left(1+\delta^{\sigma} \beta^{\sigma} R^{\sigma-1}\right)}
$$

For $\sigma<1, \vartheta(\beta)$ is a decreasing function of $\beta$ when $\beta<1$ with $\vartheta(1)>1$. Therefore, $\vartheta(\beta)>1$.

For $\sigma>1, \vartheta(\beta)$ is an increasing function of $\beta$ when $\beta<1$ with $\vartheta(0)=1$. Therefore, $\vartheta(\beta)>1$. Finally, in any case, $\vartheta(\beta)>1$ and $Z^{\sigma}-(\beta \delta)^{\sigma}>0$.

Coming back to condition (11), it remains to prove that this condition holds when $K<\xi<M$. A sufficient condition for that is:

$$
K=\frac{C}{R^{\sigma} Z^{\sigma}(R \beta \delta)^{\sigma}}\left[1+R^{\sigma-1} Z^{\sigma}+R^{\sigma-1}(\beta \delta)^{\sigma}\right]>C \frac{1+R^{\sigma-1}(\beta \delta)^{\sigma}}{R^{\sigma-1}\left[Z^{\sigma}-(\beta \delta)^{\sigma}\right]}
$$

After some calculations, taking into account the expression of $Z$ given by (5), this inequality is equivalent to $\beta<1$ which is true by assumption.

This technical lemma allows to conclude on the optimal behavior of self 1.

6. Proof of proposition 4: solution in the case $K<A+B / R<L$ and $(R \beta \delta)^{\sigma} A<B$.

In this case, it is not possible to reach the solution $x=\check{x}, y=0$ associated with $(\check{c}, \check{d}, \check{e})$. Therefore, the equilibrium of the game between selves 1 and 2 is obtained by the comparison between $U(\hat{c}, \hat{d}, \hat{e})$ and $U(A, B, C)$. The last value of the utility corresponds to the solution $x=0$ and $y=0$.

Before doing this comparison, it is useful to note that the indirect utility

$$
U(\check{c}, \check{d}, \check{e})=\frac{\sigma}{\sigma-1}\left(A+\frac{B}{R}\right)^{1-\frac{1}{\sigma}}\left[1+R^{\sigma-1}(\beta \delta)^{\sigma}\right]^{\frac{1}{\sigma}}+\frac{\sigma}{\sigma-1} \beta \delta^{2}(C)^{1-\frac{1}{\sigma}}
$$

can also be interpreted as the utility that self 1 could get in the absence of the constraint $x \geq 0$ (but with the constraint $y \geq 0$ which is binding). Consequently, a first property is obtained: $U(\check{c}, \check{d}, \check{e})>U(A, B, C)$. With no constraint on $x$, it would be optimal to have a negative amount of savings in period 1 as $(R \beta \delta)^{\sigma} A<B$. 
In the case $M \leq A+B / R<L$, it is easy to conclude. Indeed, it is known that $U(\hat{c}, \hat{d}, \hat{e}) \geq U(\check{c}, \check{d}, \check{e})$ and $U(\check{c}, \check{d}, \check{e})>U(A, B, C)$. Consequently $U(\hat{c}, \hat{d}, \hat{e})>U(A, B, C)$. The game between selves 1 and 2 leads to the decisions $x=\hat{x}$ and $y=Y(\hat{x})$. This proves the part 1 of proposition (4).

The case $K<A+B / R<M$ needs a particular study as $U(\hat{c}, \hat{d}, \hat{e})<$ $U(\check{c}, \check{d}, \check{e})$ and $U(\check{c}, \check{d}, \check{e})>U(A, B, C)$. Thus, it remains to compare $U(\hat{c}, \hat{d}, \hat{e})$ with $U(A, B, C)$. This comparison is made through the following lemma:

Lemma 4 Assume that $C>B(R \beta \delta)^{\sigma},(R \beta \delta)^{\sigma} A<B$ and $K<A+B / R<$ $M$. Let us consider a given value of $C$ and a given value of $\xi=A+B / R$ with $K<\xi<M$. A and $B$ may vary in such a way that $\xi=A+B / R$, with a higher bound $A^{h}(\xi)$ for $A$ such that $A^{h}(\xi)=\xi /\left[1+R^{\sigma-1}(\beta \delta)^{\sigma}\right]$ and a lower bound $A^{l}(\xi)$ for $A$ such that $A^{l}(\xi)=\xi-C /\left[R(R \beta \delta)^{\sigma}\right]$. There exists an increasing function $\alpha(\xi)$ that satisfies $A^{l}(\xi)<\alpha(\xi)<A^{h}(\xi)$ and such that

- for $A^{l}(\xi)<A<\alpha(\xi), U(\hat{c}, \hat{d}, \hat{e})>U(A, B, C)$ : the game between selves 1 and 2 leads to the decisions $x=\hat{x}$ and $y=Y(\hat{x})$.

- for $\alpha(\xi)<A<A^{h}(\xi), U(\hat{c}, \hat{d}, \hat{e})<U(A, B, C)$ : the game between selves 1 and 2 leads to the decisions $x=0$ and $y=0$.

- for $A=\alpha(\xi)$, self 1 is indifferent between these two solutions.

The function $\alpha(\xi)$ is implicitly defined by:

$$
u(\alpha)+\beta \delta u[R(\xi-\alpha)]+\beta \delta^{2} u(C)=U(\hat{c}, \hat{d}, \hat{e})
$$

Proof. Let us consider a given value of $C$ and a given value of $\xi=A+B / R$ with $K \leq \xi<M$. $U(\hat{c}, \hat{d}, \hat{e})$ and $U(\check{c}, \check{d}, \check{e})$ only depend on $\xi$ and $C$ and they are fixed. For this given value of $\xi$, it is possible to consider different values for $A$ and $B$ such that $\xi=A+B / R$. The highest possible value of $A$ is such that $(R \beta \delta)^{\sigma} A=B$, and it corresponds to the lowest possible value for $B$. This gives the values

$$
\begin{aligned}
A^{h}(\xi) & =\frac{\xi}{1+R^{\sigma-1}(\beta \delta)^{\sigma}} \\
B^{l}(\xi) & =\frac{(R \beta \delta)^{\sigma} \xi}{1+R^{\sigma-1}(\beta \delta)^{\sigma}}
\end{aligned}
$$

For $A=A^{h}(\xi)$ and $B=B^{l}(\xi), U(\check{c}, \check{d}, \check{e})=U(A, B, C)$. Indeed the optimal choice of $x$ without any constraint for $x$ (and $y=0$ ) is such that $x=0$. As $U(\hat{c}, \hat{d}, \hat{e})<U(\check{c}, \check{d}, \check{e})$ (and $\xi<M)$, it is clear that $U(\hat{c}, \hat{d}, \hat{e})<U(A, B, C)$. The game between selves 1 and 2 leads to the decisions $x=0$ and $y=0$. 
The highest possible value of $B$ is such that $C=B(R \beta \delta)^{\sigma}$, and it corresponds to the lowest value of $A$. This gives the values

$$
\begin{aligned}
A^{l}(\xi) & =\xi-\frac{C}{R(R \beta \delta)^{\sigma}} \\
B^{h}(\xi) & =\frac{C}{(R \beta \delta)^{\sigma}}
\end{aligned}
$$

For $B$ such that $C=B(R \beta \delta)^{\sigma}, \tilde{x}=0$. The objective function of self one is concave on $(0,+\infty)$. The optimal choice of $x$ again is given by (6). It is positive as

$$
R^{\sigma} Z^{\sigma} A-B-\frac{C}{R}>0
$$

Indeed, for $A=A^{l}(\xi)$ and $B=B^{h}(\xi)$, this inequality becomes:

$$
\xi>\frac{C}{R^{\sigma} Z^{\sigma}(R \beta \delta)^{\sigma}}\left[1+R^{\sigma-1} Z^{\sigma}+R^{\sigma-1}(\beta \delta)^{\sigma}\right]=K
$$

which is true as $\xi>K$ by assumption. Therefore, in this case, $U(\hat{c}, \hat{d}, \hat{e})$ corresponds to the optimal choice of self 1 and $U(\hat{c}, \hat{d}, \hat{e})>U(A, B, C)$.

Finally, the expression of $U(A, B, C)$ is studied as a function of $A$ and $\xi$ :

$$
G(A, \xi) \equiv u(A)+\beta \delta u[R(\xi-A)]+\beta \delta^{2} u(C)
$$

The derivative $\partial G(A, \xi) / \partial x$ is positive as it leads to

$$
A^{-\frac{1}{\sigma}}-R \beta \delta B^{-\frac{1}{\sigma}}>0 \Leftrightarrow(R \beta \delta)^{\sigma} A<B
$$

$G(A, \xi)$ is an increasing function of $A$. $G$ is such that $\left.G\left(A^{l}(\xi), \xi\right)\right)<U(\hat{c}, \hat{d}, \hat{e})$ and $G\left(A^{h}(\xi), \xi\right)>U(\hat{c}, \hat{d}, \hat{e})$. Therefore, the existence and uniqueness of $\alpha(\xi)$ is proved and

- for $A^{l}(\xi)<A<\alpha(\xi), U(\hat{c}, \hat{d}, \hat{e})>U(A, B, C)$ : the game between selves 1 and 2 leads to the decisions $x=\hat{x}$ and $y=Y(\hat{x})$.

- for $\alpha(\xi)<A<A^{h}(\xi), U(\hat{c}, \hat{d}, \hat{e})<U(A, B, C)$ : the game between selves 1 and 2 leads to the decisions $x=0$ and $y=0$.

- for $A=\alpha(\xi), U(\hat{c}, \hat{d}, \hat{e})=U(A, B, C)$.

It remains to prove that $\alpha(\xi)$ is an increasing function. The function $\alpha(\xi)$ is implicitly defined by: $U(A, R(\xi-\alpha), C)=U(\hat{c}, \hat{d}, \hat{e})$, or

$\frac{\sigma}{\sigma-1}\left[(\alpha)^{1-\frac{1}{\sigma}}+(R(\xi-\alpha))^{1-\frac{1}{\sigma}}+(C)^{1-\frac{1}{\sigma}}\right]=\frac{\sigma}{\sigma-1}\left(\xi+\frac{C}{R^{2}}\right)^{1-\frac{1}{\sigma}}\left[1+R^{\sigma-1} Z^{\sigma}\right]^{\frac{1}{\sigma}}$ 
The derivative $\alpha^{\prime}(\xi)$ is such that:

$$
\left[A^{-\frac{1}{\sigma}}-R \beta \delta B^{-\frac{1}{\sigma}}\right] \alpha^{\prime}(\xi)=\left(\xi+\frac{C}{R^{2}}\right)^{-\frac{1}{\sigma}}\left[1+R^{\sigma-1} Z^{\sigma}\right]^{\frac{1}{\sigma}}-R \beta \delta B^{-\frac{1}{\sigma}}
$$

The term $A^{-\frac{1}{\sigma}}-R \beta \delta B^{-\frac{1}{\sigma}}$ is positive as by assumption $(R \beta \delta)^{\sigma} A<B$. It remains to prove that the right-hand side is positive. To prove that, it is first proved that

$$
\left(\xi+\frac{C}{R^{2}}\right)^{-\frac{1}{\sigma}}\left[1+R^{\sigma-1} Z^{\sigma}\right]^{\frac{1}{\sigma}}>A^{-\frac{1}{\sigma}}
$$

this inequality is equivalent to

$$
A\left[1+R^{\sigma-1} Z^{\sigma}\right]>A+\frac{B}{R}+\frac{C}{R^{2}}
$$

which gives $A R^{\sigma-1} Z^{\sigma}>B+C / R$. This last inequality is true. Indeed, the inequality $K<A+B / R$ leads to

$$
A+\frac{B}{R}>\frac{C}{R^{\sigma} Z^{\sigma}(R \beta \delta)^{\sigma}}\left[1+R^{\sigma-1} Z^{\sigma}+R^{\sigma-1}(\beta \delta)^{\sigma}\right]
$$

or

$$
A R^{\sigma} Z^{\sigma}>-\frac{B R^{\sigma} Z^{\sigma}}{R}+\frac{C}{R}+\frac{C}{(R \beta \delta)^{\sigma}}\left[1+R^{\sigma-1} Z^{\sigma}\right]
$$

By assumption, $C>B(R \beta \delta)^{\sigma}$, which allows us to write:

$$
A R^{\sigma} Z^{\sigma}>-\frac{B R^{\sigma} Z^{\sigma}}{R}+\frac{C}{R}+B\left[1+R^{\sigma-1} Z^{\sigma}\right]
$$

that gives $A R^{\sigma-1} Z^{\sigma}>B+C / R$. Finally, it has been proved that

$$
\left(\xi+\frac{C}{R^{2}}\right)^{-\frac{1}{\sigma}}\left[1+R^{\sigma-1} Z^{\sigma}\right]^{\frac{1}{\sigma}}>A^{-\frac{1}{\sigma}}
$$

As it is assumed that $A^{-\frac{1}{\sigma}}>R \beta \delta B^{-\frac{1}{\sigma}}$, it implies that

$$
\left(\xi+\frac{C}{R^{2}}\right)^{-\frac{1}{\sigma}}\left[1+R^{\sigma-1} Z^{\sigma}\right]^{\frac{1}{\sigma}}>R \beta \delta B^{-\frac{1}{\sigma}}
$$

Finally, $\alpha$ is an increasing function of $\xi$.

Using lemma (4), it is possible to prove the second part of proposition (4). 
From the relation $A=\alpha(\xi)$, it is possible to find a relation between $A$ and $B$ given by: $B=R\left[\alpha^{-1}(A)-A\right] \equiv \Delta(A)$. It is easy to show that $\Delta(A)$ is a decreasing function. Indeed, $\Delta(A)$ is implicitly defined by $U(A, B, C)=$ $U(\hat{c}, \hat{d}, \hat{e})$, or

$\frac{\sigma}{\sigma-1}\left[(A)^{1-\frac{1}{\sigma}}+(B)^{1-\frac{1}{\sigma}}+(C)^{1-\frac{1}{\sigma}}\right]=\frac{\sigma}{\sigma-1}\left(A+\frac{B}{R}+\frac{C}{R^{2}}\right)^{1-\frac{1}{\sigma}}\left[1+R^{\sigma-1} Z^{\sigma}\right]^{\frac{1}{\sigma}}$

The derivative $\Delta^{\prime}(A)$ is such that:

$$
\begin{aligned}
& {\left[R \beta \delta B^{-\frac{1}{\sigma}}-\left(A+\frac{B}{R}+\frac{C}{R^{2}}\right)^{-\frac{1}{\sigma}}\left(1+R^{\sigma-1} Z^{\sigma}\right)^{\frac{1}{\sigma}}\right] \frac{\Delta^{\prime}(A)}{R} } \\
= & \left(A+\frac{B}{R}+\frac{C}{R^{2}}\right)^{-\frac{1}{\sigma}}\left(1+R^{\sigma-1} Z^{\sigma}\right)^{\frac{1}{\sigma}}-A^{-\frac{1}{\sigma}}
\end{aligned}
$$

From the preceding results (cf. (12) and (13)), it is known that

$$
\begin{aligned}
\left(A+\frac{B}{R}+\frac{C}{R^{2}}\right)^{-\frac{1}{\sigma}}\left(1+R^{\sigma-1} Z^{\sigma}\right)^{\frac{1}{\sigma}}-A^{-\frac{1}{\sigma}} & >0 \\
R \beta \delta B^{-\frac{1}{\sigma}}-\left(A+\frac{B}{R}+\frac{C}{R^{2}}\right)^{-\frac{1}{\sigma}}\left(1+R^{\sigma-1} Z^{\sigma}\right)^{\frac{1}{\sigma}} & <0
\end{aligned}
$$

Therefore $\Delta^{\prime}(A)<0$.

From (14), it is easy to check that

$$
\begin{aligned}
\Delta\left(A_{H}\right) & =\Delta\left(\frac{C}{(R Z)^{\sigma}(R \beta \delta)^{\sigma}}\left[1+R^{\sigma-1}(\beta \delta)^{\sigma}\right]\right)=\frac{C}{(R \beta \delta)^{\sigma}} \\
\Delta\left(A_{I}\right) & =\Delta\left(\frac{M}{1+R^{\sigma-1}(\beta \delta)^{\sigma}}\right)=\frac{M(R \beta \delta)^{\sigma}}{1+R^{\sigma-1}(\beta \delta)^{\sigma}}
\end{aligned}
$$

